\title{
A Case of Giant Colonic Muco-submucosal Elongated Polyps Associated with Intussusception
}

\author{
Joo Heon Kim · Seung Yun Lee \\ Je Ho Jang ${ }^{1}$. Hyun Young Han ${ }^{2}$ \\ Dong Wook Kang \\ Departments of Pathology, ${ }^{1}$ Surgery, and \\ ${ }^{2}$ Radiology, Eulji University Hospital, Daejeon, \\ Korea
}

Received: February 27, 2016

Revised: April 3, 2016

Accepted: April 27, 2016

Corresponding Author

Dong Wook Kang, MD

Department of Pathology, Eulji University Hospital,

95 Dunsanseo-ro, Seo-gu, Daejeon 35233, Korea

Tel: +82-42-611-3454

Fax: +82-42-611-3459

E-mail: astrias@eulji.ac.kr
Colonic muco-submucosal elongated polyp (CMSEP), a newly categorized non-neoplastic colorectal polyp, is a pedunculated and elongated polyp composed of normal mucosal and submucosal layers without any proper muscle layer. We herein report a giant variant of CMSEP associated with intussusception in the rectosigmoid colon, with a review of the literature. A 48-yearold woman underwent a laparoscopic low anterior resection due to multiple large submucosal polypoid masses associated with intussusception. Grossly, the colonic masses were multiple pedunculated polyps with a long stalk and branches ranging in size from a few millimeters to 14.0 $\mathrm{cm}$ in length. Microscopically, there was no evidence of hyperplasia, atypia, or active inflammation in the mucosa. The submucosal layers were composed of edematous and fibrotic stroma with fat tissue, dilated vessels, and lymphoid follicles.

Key Words: Non-neoplastic colorectal polyps; Colonic muco-submucosal elongated polyp; Intussusception
The term polyp in the gastrointestinal tract is a nonspecific clinical term, broadly utilized when referring to any localized elevation above the surrounding mucosa regardless of its histologic nature, and can be categorized as neoplastic and non-neoplastic polyps. The latter are traditionally classified as hyperplastic, inflammatory, and hamartomatous polyps. ${ }^{1}$ Daniels and Montgomery, ${ }^{2}$ however, differently subclassified the non-neoplastic polyps in the colorectum into mucosal prolapse, incidental stromal polyps and polyps associated with or without systemic diseases. As such variations in classification, currently there is no clear cut consensus regarding the most ideal or clinically significant classification scheme for the various non-neoplastic colorectal polyps.

Recently, we experienced a large non-neoplastic colorectal polyp which did not match the traditional classification of nonneoplastic colorectal polyps, in addition to being associated with intussusception. In this report, we present a case of a giant variant of the newly classified non-neoplastic colorectal polyp, colonic muco-submucosal elongated polyp (CMSEP), associated with intussusception in a 48-year-old woman, with a review of the literature.

\section{CASE REPORT}

A 48-year-old, previously healthy female patient visited the emergency department with initial presentation of intermittent abdominal pain and anal bleeding. The patient explained that the symptom had first begun 4 to 5 years ago. Under the clinical impression of hemorrhoids, the patient underwent a hemorrhoidectomy. The patient had no particular family history, changing bowel habits or mucoid diarrhea. An abdominal computed tomography $(\mathrm{CT})$ was conducted 2 weeks later as a part of the evaluation process, which revealed a colocolic intussusception from the sigmoid colon to the distal rectum. In addition, prominent mucosal folds were observed without any ischemic change or pericolic abnormality. Following the CT scan, a barium enema revealed a large movable and compressible submucosal mass in the distal portion of the sigmoid colon (Fig. 1). Multiple similar yet smaller lesions were also present near the main mass in the sigmoid colon. The intussusception spontaneously regressed without any complications. Colonoscopy revealed several large polypoid masses in the rectosigmoid colon and multiple biopsies were obtained. The biopsied specimens were diagnosed as chronic 

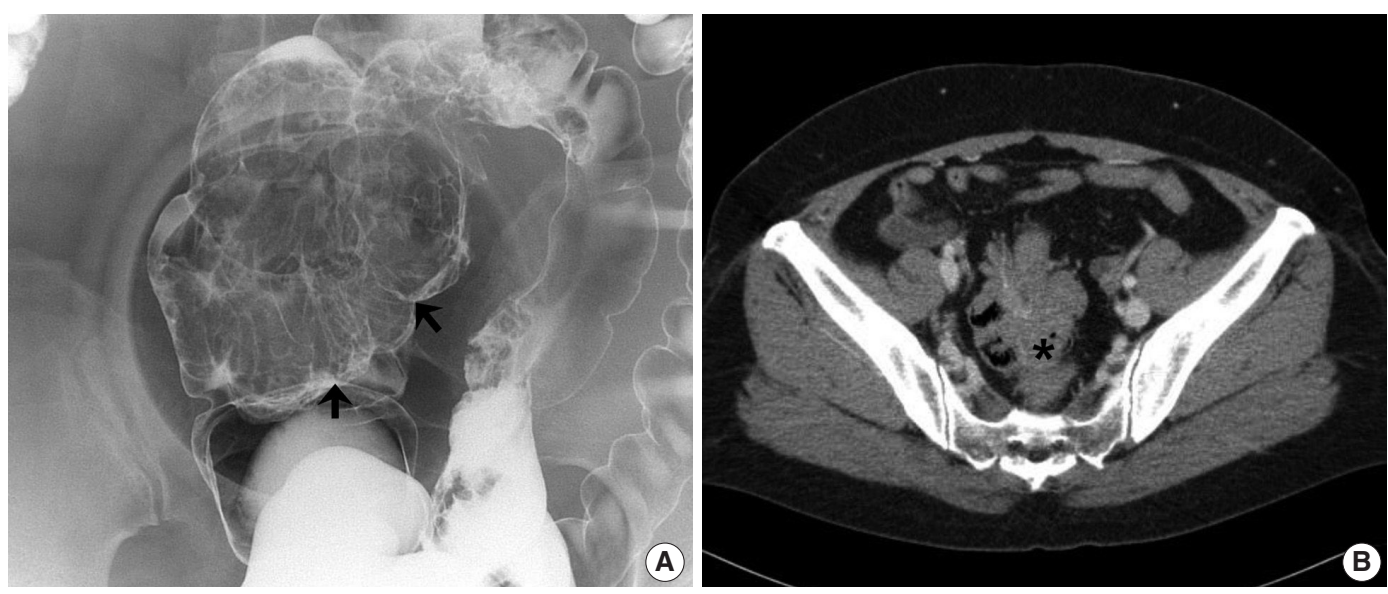

Fig. 1. Barium enema (A) and abdominal computed tomography (B) of colonic muco-submucosal elongated polyp reveal prominent mucosal and submucosal folds (arrow) associated with intussusception (asterisk) in rectosigmoid colon.
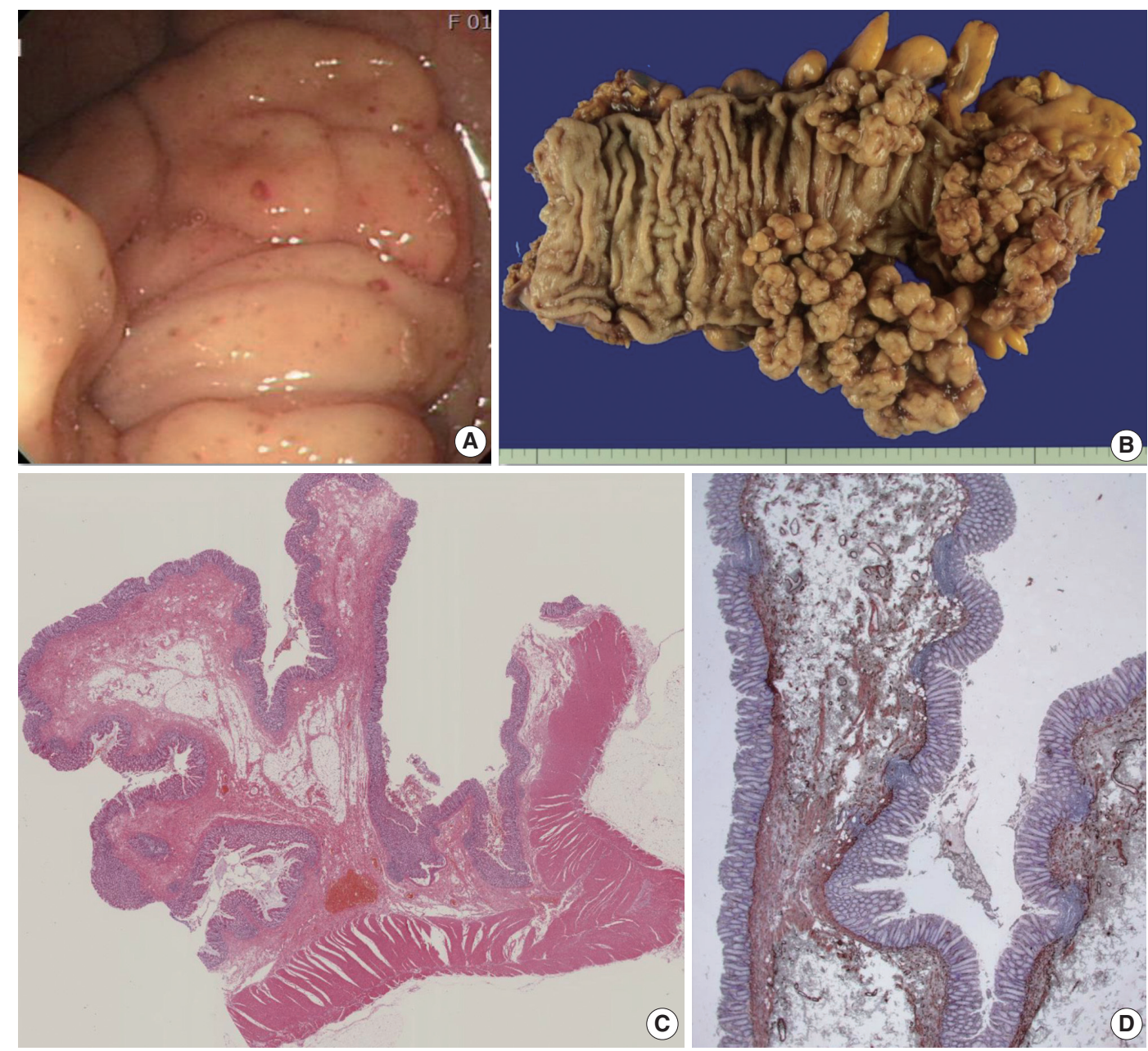

Fig. 2. Colonoscopy, gross and microscopic findings of colonic muco-submucosal elongated polyp. (A) Colonoscopy reveals multiple, large, movable, pedunculated, and sessile polyps from descending to sigmoid colon. (B) The polypoid masses show large, tree-like branching mucosal folds with elongated and pedunculated stalks with a broad base. (C) The polyps have long, elongated, and loose submucosal elongated stalks without cytological atypia and active inflammation. (D) $\alpha$-Smooth muscle actin immunohistochemical stain show prominent smooth muscle of the muscularis mucosae. 
Table 1. Review of the literature for CMSEPS

\begin{tabular}{|c|c|c|c|c|c|}
\hline Reference (published year) & Country & $\operatorname{Sex}(M: F)$ & Age (mean, yr) & Site (number of polyps) & Polyp size (length, $\mathrm{cm}$ ) \\
\hline Iveković et al..$^{17}(2015)$ & Croatia & $M$ & 82 & SC (1) & 2.4 \\
\hline Tan et al. ${ }^{16}$ (2013) & Singapore & $1: 2$ & 66.3 & SI (2), DC (1) & $1.4-4.0$ \\
\hline Shin et al. ${ }^{18}(2012)$ & Korea & M & 54 & $\mathrm{AC}(1)$ & 3.5 \\
\hline Nishimura et al..$^{8}$ (2012) & Japan & $\mathrm{F}$ & 70 & $\mathrm{SI}(1)$ & NS \\
\hline Hirasaki et al. ${ }^{5}$ (2011) & Japan & M & 79 & SC (1) & 0.7 \\
\hline Ambrosio et al. ${ }^{13}$ (2011) & Italy & $\mathrm{F}$ & 77 & SC (1) & 7.0 \\
\hline Alizart et al.12 (2011) & Australia & $7: 4$ & 57.6 & SC (9), TC (2), AC (1), DC (1) & $1.0-15.0$ \\
\hline Tozawa et $a l . .^{10}(2009)$ & Japan & M & 31 & $\mathrm{DC}(1)$ & 2.0 \\
\hline Kume et al. ${ }^{7}$ (2009) & Japan & M & 66 & $\mathrm{TC}(1)$ & 4.0 \\
\hline Kanazawa et al. ${ }^{6}$ (2006) & Japan & M & 53 & $A C(1)$ & 5.0 \\
\hline Akahoshi et al. ${ }^{4}$ (2005) & Japan & $5: 2$ & 63.6 & SC (2), AC (2), R (3) & $1.0-4.0$ \\
\hline Yamamoto et al. ${ }^{11}$ (2004) & Japan & M & 73 & $\mathrm{DC}(1)$ & NS \\
\hline Lee et al..$^{15}$ (2003) & Korea & M & 44 & $A C(1)$ & 6.0 \\
\hline Takahashi et al. ${ }^{9}$ (2002) & Japan & M & 52 & $\mathrm{TC}(1)$ & 1.0 \\
\hline Domoto et al..$^{14}$ (1998) & Japan & $\mathrm{F}$ & 55 & $\mathrm{DC}(1)$ & 3.0 \\
\hline Matake et al. ${ }^{3}$ (1998) & Japan & $6: 9$ & 58.3 & TC (6), AC (4), DC (2), SC (3) & $0.9-16.0$ \\
\hline Total & & $28: 20$ & 60.2 & 48 cases $(50)$ & $0.7-16.0$ \\
\hline
\end{tabular}

CMSEP, colonic muco-submucosal elongated polyps; M, male; F, female; SC, sigmoid colon; SI, small intestines; DC, descending colon; AC, ascending colon; NS, not specified; TC, transverse colon; $R$, rectum.

nonspecific inflammation without any definite submucosal masslike lesions. Due to the presence of such large lesions, the patient underwent a laparoscopic low anterior resection in which a 21.5-cm segment of colon was resected.

The surgical specimen revealed multiple, large, tree-like branching polypoid masses, with elongated or pedunculated stalks or a wide broad base, measuring up to $14.0 \times 5.0 \times 4.5 \mathrm{~cm}$ in dimensions of the largest polypoid mass (Fig. 2A). Upon microscopic examination of the lesions, most of the polyps had long, elongated, and loose or fibrotic submucosal elongated stalks, while some were sessile. There was neither prominent cytologic atypia nor active inflammation in the mucosa. There was also proliferation of mature fat tissue, lymphoid follicles, multiple dilated blood vessels, and hypertrophied smooth muscle of the muscularis mucosae (Fig. 2B). Immunohistochemical staining with $\alpha$-smooth muscle actin revealed hypertrophied smooth muscle of the muscularis mucosae (Fig. 2C). However, there was no tree like proliferation of the muscularis mucosae as in Peutz-Jeghers polyps. Staining with other antibodies, such as anaplastic lymphoma kinase, c-Kit, $\beta$-catenin, CD34, and S-100 protein, showed negative findings in all.

\section{DISCUSSION}

The newly categorized non-neoplastic colorectal polyp, CMSEP, was first described by Matake et $a l^{3}{ }^{3}$ in 1998. Since its first appearance in the literature, there have been various reports of such polyps, although mostly from Japanese institutions. ${ }^{3-11}$
The characteristic endoscopic feature is the "worm-like" appearance. Histologically, the polyps are covered by normal mucosa and a loose to dense submucosal layer containing a variably prominent mixture of blood vessels and lymphatics, in the absence of significant active inflammation. Such polyps have been reported in all parts of the large intestines, as well as in the small intestines. ${ }^{3-5,7-17}$ Overall, the histologic findings of our case are similar to those of previously reported cases, and our case meets the suggested criteria for the diagnosis of CMSEP by Alizart $e t$ al..$^{12}$ and Tan et al. ${ }^{16}$

In the literature to date, a total of 48 cases of CMSEPs have been reported (Table 1). ${ }^{3-18}$ Based on the review of these reports, there seems to be a slight predilection for males (male:female = 1.4:1). All but one of these cases ${ }^{12}$ were a single polyp occurring in the colon, and the sigmoid colon was the most common site. ${ }^{3-5,12,13}$ Yet, our case is unique in that there was the additional presentation of intussusception and in that the polyps were multiple and much larger in size compared to the majority of the previously reported cases. Furthermore, there has been no report to date of such large polyps in the rectosigmoid colon displaying branching architecture as in our case. The mean size of the reported polyps in the sigmoid colon and rectum was $2.44 \mathrm{~cm}$ and $1.12 \mathrm{~cm}$, respectively, which is much smaller compared to the polyps in this report that measured up to $16.0 \mathrm{~cm}$ in largest dimensions. Despite the multiplicity and large size, the histologic features of our case match the descriptions of CMSEPs.

Many differential diagnoses must be considered in the diagnosis of CMSEP. The first differential diagnosis includes mucosal 
prolapse polyps in patients with mucosal prolapse syndrome (MPS). MPS comprises a variety of clinico-pathologic entities with mucosal prolapse as the underlying pathogenic mechanism., ${ }^{2,6}$ Solitary rectal ulcer syndrome (SRUS) is the most common disorder of MPS. Gross and microscopic findings of SRUS show solitary or multiple ulcerated or polypoid lesions with crypt hyperplasia and villiform or serrated change and characteristic fibromuscular proliferation in the lamina propria. $^{2}$ However, our case does not match SRUS clinically and pathologically. Another differential diagnosis to be considered is filiform polyposis which is characterized by numerous long slender "worm-like" projections in patients with inflammatory bowel disease. ${ }^{2}$ Filiform polyposis is generally thought to be a post-inflammatory reparative process of inflammatory bowel disease, and it is also referred to as inflammatory polyposis or pseudopolyposis. There have been reported cases of giant filiform polyposis associated with inflammatory bowel disease. ${ }^{19}$ Although the overall shape of filiform polyps is similar to that of the presenting case, the patient did not exhibit any evidence of inflammatory bowel disease. Hamartomatous polyps are also included in the differential diagnosis due to the presence of proliferation of submucosal components such as blood vessels, hypertrophied muscularis mucosae, and mature adipose tissue. Peutz-Jegher polyps present hypertrophied branching frameworks of smooth muscles of the muscularis mucosae and the Cronkhite-Canada polyps reveal cystically dilated mucus glands with inflamed edematous stroma. ${ }^{1,2}$ However, there are no branching frameworks of muscularis mucosae and active inflammation in our case. The possibilities of ganglioneuroma, inflammatory myofibrobastic tumor, inflammatory fibroid polyp, and desmoid tumor could also be ruled out.

The exact pathogenesis of CMSEPs is currently unknown. Some suggest that CMSEPs show the clinico-pathologic features of mucosal prolapse polyps and the peristaltic movement of the gastrointestinal tract serves as a mechanical traction for redundant mucosa, in which such areas of the mucosa become the starting point of polyp formation. ${ }^{2}$ Alizart et al. ${ }^{12}$ proposed that focal areas with prominent submucosal venous plexus may elevate the mucosa, thus becoming the leading point for the traction and ultimately leading to the polyp formation. Kelly ${ }^{20}$ presented a similar explanation for the formation of polypoid prolapsing mucosal folds in diverticular disease. However, we suggest that a combination of both mucosal prolapse and a hamartomatous process may produce a giant variant of CMSEP.

The CMSEP is a non-neoplastic polyp with a unique characteristic "worm-like" appearance. Due to the polyps being covered by normal mucosa and submucosal layers, at times, the superfi- cially obtained endoscopic biopsy specimen of CMSEP may be diagnosed differently, depending on the pathologist, as a hyperplastic polyp or even chronic nonspecific inflammation, in the absence of any endoscopic information. Thus, endoscopic findings along with the microscopic findings should be carefully considered in reaching the diagnosis of CMSEP.

\section{Conflicts of Interest}

No potential conflict of interest relevant to this article was reported.

\section{REFERENCES}

1. Kumar V, Abbas AK, Fausto N, Aster JC. Robbins and Cotran pathologic basis of disease. 8th ed. Philadelphia: Saunders Elsevier, 2010; 815-22.

2. Daniels J, Montgomery E. Non-neoplastic colorectal polyps. Curr Diagn Pathol 2007; 13: 467-78.

3. Matake H, Matsui T, Yao T, et al. Long pedunculated colonic polyp composed of mucosa and submucosa: proposal of a new entity, colonic muco-submucosal elongated polyp. Dis Colon Rectum 1998; 41: 1557-61.

4. Akahoshi K, Matsumoto M, Kimura M, et al. Colonic muco-submucosal elongated polyp: diagnosis with endoscopic ultrasound. Br J Radiol 2005; 78: 419-21.

5. Hirasaki S, Koide N, Fukazawa M, Fujiwara K. Small muco-submucosal elongated polyp of the sigmoid colon. Intern Med 2011; 50: 1855.

6. Kanazawa N, Kawahara Y, Kasahara I, Higuchi Y, Arai T, Kuroiwa K. Polypoid prolapsing mucosal folds with diverticulosis and colonic muco-submucosal elongated polyps. Geriatr Gerontol Int 2006; 6: 65-8.

7. Kume K, Sakata H, Yoshikawa I, Watanabe T, Harada M. Polypectomy of a colonic muco-submucosal elongated polyp. Endoscopy 2009; 41 Suppl 2: E265-6.

8. Nishimura J, Nishikawa J, Tanabe R, et al. A case of enteric mucosubmucosal elongated polyp. Nihon Shokakibyo Gakkai Zasshi 2012; 109: 1940-5.

9. Takahashi M, Kubokawa M, Tanaka M, et al. Endoscopic ultrasonography features of colonic muco-submucosal elongated polyp. Endoscopy 2002; 34: 515.

10. Tozawa K, Ohda Y, Matsumoto T, Hori K, Nishigami T. Clinical challenges and images in GI: colonic mucosubmucosal elongated polyp. Gastroenterology 2009; 136: 49, 366.

11. Yamamoto T, Ishii T, Kawakami T, et al. Colonic muco-submucosal 
elongated polyp. Gastrointest Endosc 2004; 59: 868.

12. Alizart MM, Rosty C, Brown IS. Colonic mucosubmucosal elongated polyp: a clinicopathologic study of 13 cases and review of the literature. Am J Surg Pathol 2011; 35: 1818-22.

13. Ambrosio MR, Rocca BJ, Ginori A, Barone A, Onorati M, Lazzi S. Long pedunculated colonic polyp with diverticulosis: case report and review of the literature. Pathologica 2011; 103: 8-10.

14. Domoto H, Terahata S, Sakai Y, Katoh S, Iwai A, Itoh K. A case of colonic muco-submucosal elongated polyp with ischemic colitis as a first diagnostic clue. Nihon Shokakibyo Gakkai Zasshi 1998; 95: 777-80.

15. Lee HS, Choi BK, Seo WS, et al. A case of colonic muco-submucosal elongated polyp. Korean J Gastrointest Endosc 2003; 26: 103-5.

16. Tan CL, Tan SH, So JB, Petersson F. Muco-submucosal elongated polyps of the gastrointestinal tract: a case series and a review of the literature. World J Gastroenterol 2013; 19: 1845-9.

17. Iveković MP, Radulović B, Brćić I, Jakic Razumović J, Rustemović $\mathrm{N}$, Iveković $\mathrm{H}$. Colonic muco-submucosal elongated polyp: a case report and literature review. Lijec Vjesn 2015; 137: 168-70.

18. Shin Y, Kim JB, Choi JS, et al. A case of nonpolypoid cancer arising from colonic muco-submucosal elongated polyp. Korean J Gastroenterol 2012; 59: 257-9.

19. Ponte R, Mastracci L, Di Domenico S, et al. Giant filiform polyposis not associated with inflammatory bowel disease: a case report. Viszeralmedizin 2015; 31: 58-60.

20. Kelly JK. Polypoid prolapsing mucosal folds in diverticular disease. Am J Surg Pathol 1991; 15: 871-8. 